\title{
Les rouages de la guerre contemporaine
}

\section{Christophe Wasinski}

\section{(2) OpenEdition}

1 Journals

\section{Édition électronique}

URL : http://journals.openedition.org/conflits/18691

DOI : 10.4000/conflits. 18691

ISSN : 1777-5345

Éditeur :

CECLS - Centre d'études sur les conflits - Liberté et sécurité, L'Harmattan

\section{Édition imprimée}

Date de publication : 15 juin 2013

Pagination : 135-140

ISBN : 978-2-343-01116-5

ISSN : 1157-996X

\section{Référence électronique}

Christophe Wasinski, «Les rouages de la guerre contemporaine », Cultures \& Conflits [En ligne], 89 | printemps 2013, mis en ligne le 15 juin 2013, consulté le 31 mars 2021. URL : http://

journals.openedition.org/conflits/18691 ; DOI : https://doi.org/10.4000/conflits.18691

Ce document a été généré automatiquement le 31 mars 2021.

Creative Commons License 


\title{
Les rouages de la guerre contemporaine
}

\author{
Christophe Wasinski
}

\section{RÉFÉRENCE}

Caroline M. Croser, The New Spatiality of Security: Operational Uncertainty and the US Military in Iraq, Londres et New York, Routledge, coll. "Critical Security Studies", 2010 James Der Derian, Virtuous War: Mapping the Military-Industrial Media-Entertainment Network, Londres et New York, Routledge, 2009 ( $2^{\mathrm{e}}$ édition)

Mark Duffield, Global Governance and the New Wars: The Merging of Development and Security, Londres et New York, Zed Books, 2005

Mikkel Vedby Rasmussen, The Risk Society at War: Terror, Technology and Strategy in the Twenty-First Century, Cambridge, Cambridge University Press, 2006

Martin Shaw, The New Western Way of War: Risk Transfer and its Crisis in Iraq, Cambridge, Polity Press, 2006

1 Les cinq ouvrages que nous présentons ici apportent des éclairages particulièrement bienvenus concernant la problématique des guerres contemporaines. Certes, ces textes abordent la question sous des angles variés. Les travaux de Caroline M. Croser et James Der Derian s'inspirent d'auteurs habituellement qualifiés de poststructuralistes, tels que Gilles Deleuze, Michel Foucault ou encore Paul Virilio. Mikel Vedby Rasmussen s'appuie largement sur la notion sociologique de risque développée par Ulrich Beck. Mark Duffield s'intéresse aux structures économiques internationales et aux représentations politiques pour développer un point de vue de type sociologique critique. Martin Shaw développe une sociologie de la guerre portant sur les effets concrets des conflits modernes. En d'autres termes, il existe des similarités avérées entre les ouvrages, des références croisées se retrouvent dans leurs bibliographies et la plupart de ces études prennent en considération les interrogations critiques et constructivistes sur les représentations guerrières et leurs effets. Pourtant, l'échantillon rassemblé ne constitue pas une école de pensée ou une approche 
théorique unifiée. Toutefois, et c'est probablement plus important, la juxtaposition de ces travaux souligne une sensibilité commune et met en évidence un fil conducteur. Sans permettre une compréhension exhaustive de la guerre, tous exposent avec justesse certains des rouages essentiels au maintien et à l'adaptation des capacités belliqueuses dans notre monde ${ }^{1}$.

2 Parmi ces auteurs, Mark Duffield est certainement celui qui pratique le plus la montée en généralité. Global Governance and the New Wars est une tentative d'éclairage général des nouveaux conflits violents. L'ouvrage débute en décrivant le mouvement du système mondial capitaliste. Ce dernier est marqué par une dynamique d'expansion et d'inclusion qui subit un coup d'arrêt au cours des années 1970. Le cœur de l'économie mondiale est alors composé de l'Amérique du Nord, de l'Europe et de certaines parties d'Asie. La majorité des pays du Sud ne parviennent cependant plus à s'intégrer à ce noyau. De cette exclusion économique internationale résultent de nombreuses formes d'instabilité étatique, en particulier en Afrique. Partant, les États du Nord ont commencé à représenter la pauvreté comme un facteur de danger, phénomène qui prend de l'ampleur à la fin de la guerre froide. La réponse au sous-développement ne se conçoit donc plus en termes d'aides socio-économiques et politiques mais en termes sécuritaires, techniques et apolitiques. Mark Duffield approfondit cette question en démontant les représentations qui matérialisent ce changement de vision. Il met en exergue l'existence d'une idéologie humanitaire et d'un discours sur les «nouveaux barbares ». Dans le sillage de ces évolutions, il affirme également que la gestion des désordres du Sud est prise en charge par un complexe stratégique impliquant des forces armées, des sociétés de sécurité privées, des organisations non gouvernementales, des organisations internationales et des multinationales profitant de cette situation. Ainsi, loin de résoudre durablement les problèmes des États exclus, la gouvernance de sécurité contribue au maintien des États du Sud en périphérie du système. Plus encore, de la mise sous pression des États du sud résultent des formes d'adaptation toujours plus déstabilisatrices. On assiste à la réintégration économique du Sud par des canaux détournés, par le marché noir, par les trafics en tous genres (or, diamants, bois exotiques, ou encore drogues). Et, par ce biais, il y a finalement criminalisation. En conclusion, Mark Duffield produit une carte conceptuelle des guerres qui secouent la planète depuis plusieurs décennies. Un point fort de son analyse est d'intégrer dans son explication la structure économique mondiale, la nébuleuse d'acteurs concernés par les conflits et les discours influençant les acteurs et participant à la reproduction de la structure susmentionnée. Un léger regret demeure ; Mark Duffield ne développe pas les conflits interétatiques plus classiques. Certains éléments semblent pourtant montrer que son modèle est applicable dans ce champ également (Saddam Hussein s'accaparant l'État est vaincu suite à une guerre «classique » en 2003) et que la logique des «nouvelles guerres » n'est probablement pas complètement déconnectée de celle des autres conflits.

3 Avec son livre The Risk Society at War, Mikkel Vedby Rasmussen précise la nature des rouages guerriers en se focalisant sur la charnière politique-stratégique. Cette étude est particulièrement intéressante car elle explique, à la jonction entre le décideur politique et le militaire, la mise en place d'une représentation de l'environnement international de sécurité, l'élaboration de recettes jugées opportunes pour agir dans cet environnement, et l'acquisition de la confiance accordée à l'outil militaire. Le point de départ de l'auteur est de montrer dans quelle mesure une représentation de la violence 
s'est historiquement constituée en tant que moyen rationnel pour contrer des menaces à partir d'une relecture de la pensée stratégique du passé. Avec ce que l'on a appelé la Révolution dans les Affaires Militaires et la "Transformation", concepts qui apparaissent dans les années 1990 et 2000, la croyance en la rationalité et en l'efficacité de l'outil militaire s'est encore renforcée parmi les hommes politiques. Il en résulte une conception de la guerre normalisée, bureaucratisée à l'extrême et, en dernière instance, fortement dépolitisée. Plus encore, l'outil militaire moderne est en définitive appréhendé comme un moyen de gestion préventive de l'ordre international. La guerre ne vise plus un ennemi ciblé mais plutôt des risques contre lesquels le principe de précaution s'impose. Tel serait le nouveau calcul stratégique d'après Mikkel Rasmussen. L'auteur souligne également à quel point cette représentation engendre des effets inattendus (ce qu'il nomme, à la suite d'Ulrich Beck, des effets boomerang). L'un d'entre eux, perceptible aux États-Unis en particulier, est le fait que plus les équipements militaires deviennent performants, plus la crainte de l'acquisition de ces équipements par un adversaire potentiel se renforce, ce qui encourage une quête sans fin de l'avantage technologique. De même, la modernisation des équipements donne le sentiment que la guerre ne peut plus faire de victimes parmi les civils. En conséquence, plus on dote les militaires d'armes sophistiquées, plus l'intolérance aux victimes est importante. En retour, la liberté d'action des soldats en est limitée. Ces quelques remarques n'épuisent incontestablement pas la richesse de l'ouvrage (qui développe par exemple beaucoup de commentaires fort intéressants sur la lutte contre terrorisme transnational), l'essentiel étant la tentative de cerner la façon dont la guerre se conçoit à la charnière entre les décideurs politique et les militaires. A l'évidence, le point de vue de l'auteur est différent de celui de Mark Duffield qui ne se focalise pas sur la variable technique militaire. Si les exemples prototypiques de conflits modernes sont les deux guerres contre l'Irak (1991 et 2003) pour Rasmussen, Mark Duffield s'appuie plutôt sur des guerres au Soudan et au Sierra Léone. Il existe donc, entre les deux auteurs (au moins) un espace à combler : celui qui montrerait comment se construit la confiance politique envers les interventions où les militaires ne recourent pas automatiquement et massivement aux moyens les plus modernes. Ou encore, comment se construit la confiance politique entre les opérations de maintien de la paix et de reconstruction et les actions de contre-insurrection.

4 Dans son ouvrage The New Spatiality of Warfare, Caroline M. Croser approfondit davantage encore la machine à produire de la violence instrumentale. Certes, la charnière politico-stratégique n'est pas complètement perdue de vue. Mais, l'essentiel de son travail est basé sur l'anthropologie appliquée au domaine militaire moderne ${ }^{2}$. Évoquer ici l'atypique analyse de James der Derian sur la "guerre vertueuse ${ }^{3}$ dans l'ouvrage éponyme Virtuous War, est nécessaire. Inhabituellement, ce dernier se présente en effet comme un "travelogue », une sorte de carnet de voyage. Il est en effet question de déplacement. Le premier chapitre est consacré à Fort Irwin, dans le désert de Mojave en Californie. Le troisième chapitre fait mention du Hohenfels Combat and Manoeuver Training Center (CMTC) en Allemagne. Enfin, il montre une exposition de matériel militaire de haute technologie, dans le Mariot World Center d'Orlando en Floride. Par ailleurs, il est interessant de noter que l'ouvrage n'est pas seulement un texte continu. Il est illustré par quelques photographies et on y retrouve d'assez longues interviews du général Wesley Clark et du futurologue du Pentagone Andy Marshall. L'autoréflexivité et l'humour sont également de mise. L'auteur se positionne sur le plan personnel et familial par rapport à son sujet d'exploration. Il se raconte 
ensuite sous les traits d'un candide au pays des merveilles technologiques militaires. Le résultat n'est pas sans ironie (voir par exemple le passage consacré aux tortues du désert que les blindés américains n'ont pas le droit d'écraser dans leur centre d'entraînement de Fort Irwin). Plus spécifiquement concernant notre propos, l'apport principal de Virtuous War est, comme le sous-titre l'indique, de cartographier l'existence d'un nouveau complexe militariste et de montrer dans quelle mesure ce complexe élabore de nouvelles conceptions guerrières. Dans ce sillage, James Der Derian réactualise l'ancien complexe militaro-industriel d'Eisenhower pour lui substituer son MIME-NET, Military-Industrial-Media-Entertainement Network, soit un réseau composé des militaires, des industriels de la défense, des médias et du secteur du délassement. La thèse est que l'action combinée de ces acteurs est à l'origine d'une conception aseptisée de la guerre et qu'elle facilite la perpétuation de la violence organisée. Les militaires s'entraînent grâce à des moyens électroniques de simulation. Ceux-ci ne donnent qu'une image édulcorée de la guerre et tendent à gommer la frontière entre le jeu vidéo et les pratiques violentes. Les simulations agissent par ailleurs comme des sortes de prophéties confirmant la possibilité d'un ennemi à combattre. Elles contribuent donc à la construction d'une forme d'altérité conflictuelle. $\mathrm{Au}$ combat, on recourt également de plus en plus à des moyens électroniques qui rendent les corps réels invisibles. Enfin, les médias ont tendance à s'aligner sur ce phénomène et à ne pas diffuser d'images trop dérangeantes sur les guerres américaines. Ainsi, la guerre vertueuse issue de la Révolution dans les Affaires Militaire est une guerre qui dissimule la mortalité. La guerre est vertueuse car elle a les atours de la réalité virtuelle de jeux vidéo.

5 L'ouvrage de Der Derian pourra être complété par celui de Caroline M. Croser sur les nouvelles conceptions spatiales des forces armées américaines en opération en Irak. Comme chez Der Derian, l'auteure élabore une analyse critique faisant suite à une étude de terrain. Son ouvrage s'appuie sur des interviews réalisées en 2005 parmi des militaires de la 1st Cavalry Division rentrés d'Irak. De James der Derian à Caroline M. Croser, on franchit progressivement la frontière entre la zone de l'arrière et la zone de combat, des simulations d'entraînement aux opérations réelles. Plus précisément, Caroline Croser s'intéresse à un système informatisé de commandement (CPOF pour Command Post of the Future) testé pour la première fois en Irak. Ce système mobile est composé d'un ordinateur et de trois écrans plats permettant de visualiser l'espace de combat sous plusieurs angles de vue et de projeter diverses informations relatives aux unités déployées. Ce système s'avère être le cœur du dispositif militaire de combat et est producteur d'une communauté militaire. Entre autres choses, les soldats synchronisent leurs actions à partir de l'image opérationnelle commune («common operating picture») que le CPOF diffuse. En outre, l'auteure indique que la matérialité des hommes se trouve confirmée par les représentations virtuelles du CPOF. En définitive, son étude met en évidence que le déploiement de la violence repose sur la mise en place d'une forme de représentation spatiale spécifique et que l'action militaire prend appui sur une conception construite de l'espace ${ }^{4}$. Elle note également que cette forme de représentation est de nature incomplète. On ne retrouvera pas dans l'imagerie informatisée du CPOF le ressenti corporel, l'odeur de la transpiration ou du vomi, ou encore le poids de l'équipement. En ce sens, on peut certainement parler d'effet déshumanisant au combat. par les auteurs évoqués jusqu'ici. De même, il insiste sur la dynamique engendrée par la 
Révolution dans les Affaires Militaires, la question de la guerre virtuelle et vertueuse, ou encore la représentation politique d'un outil militaire des plus efficaces. L'élément distinctif de son ouvrage est sa tentative généalogique de ce type de conflits, considérant la guerre du Vietnam et la guerre (souvent oubliée) des FalklandsMalouines. L'intérêt central de l'ouvrage repose plus encore sur la notion de transfert de risque («risk transfer») que l'on retrouve dans son sous-titre. A ce propos, l'auteur explique que la pratique guerrière est soumise à des contraintes énormes (qui font songer aux effets boomerangs de Mikkel Rasmussen). Au sein des États développés pris en considération, les décideurs politiques contraignent les militaires à des actions qui (idéalement) ont un impact économique limité et qui ne doivent pas causer de problèmes électoraux. En outre, elles sont dans l'obligation d'être rapides, de se dérouler loin de l'État qui intervient et, surtout, de minimiser les victimes parmi les troupes déployées. Finalement, Martin Shaw démontre que les discours (tels qu'analysés par Mikkel Rasmussen et James Der Derian) sur l'efficacité militaire et la guerre vertueuse, ne sont pas uniquement le reflet d'une réalité militaire mais aussi des pesanteurs qui agissent sur ceux qui doivent exécuter une opération militaire. Ces derniers sont contraints, dans les actions, de se conformer aux représentations de la Révolution dans les Affaires Militaires. Il en résulte non seulement un besoin de cacher ou rendre aussi peu visible que possible la mortalité militaire et civile (par le bias de politiques de communication appropriées), mais aussi de faire appel au discours humanitaire pour légitimer les actions violentes et la tendance à agir de façon parfois très brutale (avec des bombardements à distance très destructeurs) pour éviter les morts militaires. En définitive, la variable d'ajustement sur laquelle se reportent en dernière instance ces pesanteurs, sont les populations civiles (le cas des Irakiens est souligné par Martin Shaw). Ces populations feront principalement les frais des efforts de minimisation des risques des armées modernes.

7 En guise de conclusion, il convient de souligner le rôle des cinq ouvrages présentés quant à la compréhension des rouages de la guerre moderne. De concert, ces travaux prouvent la vitalité et la qualité des diverses approches critiques et sociologiques sur la question de la guerre. Enfin, ces études permettent de retenir un fait essentiel: l'activité guerrière contemporaine n'est pas naturelle en soi mais résulte de la mise en place de dispositifs sociaux nombreux, variés et complexes.

\section{NOTES}

1. On complétera utilement cet apercu par: Thomas Lindemann, Penser la guerre: L'apport des constructivistes, Paris, L'Harmattan, 2008; Theo Farrell, The Norms of War: Cultural Beliefs and Modern Conflict, Boulder et Londres, Lynne Rienner, 2005 ; Theo Farrell et Terry Terriff (eds.), The Sources of Military Change: Culture, Politics, Technology, Londres et Boulder, Lynne Rienner, 2002 ; Bradley S. Klein, Strategic Studies and World Order - The Global Politics of Deterrence, Cambridge, Cambridge University Press, 1994.

2. Une démarche qui avait déjà été pratiquée de façon particulièrement originale dans le champ de la stratégie nucléaire. Carol Cohn, "Sex and Death in the Rational World of Defense 
Intellectuals", Signs, vol. 12, n 4, été 1987, pp. 687-718; Hugh Gusterson, Nuclear Rites - A Weapons Laboratory at the End of the Cold War, Berkeley, University of California Press, 1998.

3. Un titre qui fait écho à un autre ouvrage, nettement moins critique à l'endroit de la violence militaire contemporaine: Michael Ignatieff, Virtual War: Kosovo and Beyond, New York, Metropolitan Books, 2001.

4. Sur cette question, voir également le très intéressant: Eyal Weizman, À travers les murs: L'architecture de la nouvelle guerre urbaine, Paris, La Fabrique, 2008.

\section{AUTEURS}

\section{CHRISTOPHE WASINSKI}

Christophe Wasinski est chercheur au sein du centre Recherche et Enseignement en Politique Internationale (REPI) à l'Université libre de Bruxelles. Il est également chargé de cours invité aux Universités catholique de Louvain et Saint-Louis de Bruxelles. 